\title{
Computer-Based Calibration and Uncertainty Analysis of GenCade: Description and Proof of Concept
}

by Sophie Munger

PURPOSE: This Coastal and Hydraulics Engineering Technical Note (CHETN) provides a description of a methodology for the computer-based calibration or Parameter Estimation (PE) and corresponding Uncertainty Analysis (UA) for the shoreline change model GenCade developed by the US Army Corps of Engineers (Frey et al. 2012a,b, 2014). This work demonstrates the suitability and benefits of applying the proposed PE and UA methods to GenCade studies using an idealized case as well as a simple field study as a proof of concept. This document is primarily for engineers and decision-makers interested in the implementation of PE and UA in one-line shoreline change models such as GenCade.

INTRODUCTION: GenCade is a one-dimensional model used to calculate shoreline change due to gradients in wave-driven sand transport rates. GenCade utilizes a semi-empirical formula to determine the longshore sand transport rates that need to be calibrated with known conditions for each site. The current state of the practice for GenCade studies is to manually calibrate the model parameters by trial-and-error, which is subjective, labor intensive, and lacks repeatability. In recent years with the availability of faster and less expensive computing resources, it has become more common to employ computer-based techniques to calibrate process-based numerical models. Computer-based PE is more objective and repeatable than manual calibration. Because PE requires several realizations of the model, it can also inform the user in regards to the uncertainty of those parameters.

Model error or the difference between the measurements and the calculated values is readily available following validation. However, model uncertainty, or the characterization of the range of values within which the true value is asserted to lie, is a topic that has remained unexplored in GenCade. Because the GenCade model is a simplification of complex physical nearshore processes, the model output has potential for a large degree of error and uncertainty. Quantifying model predictive uncertainty has never been attempted before with GenCade. As explained in greater detail in this document, quantification of model uncertainty is not a simple task. Ideally, one must not only consider model input uncertainty such as shoreline data and model parameters but also uncertainty pertaining to the model structure and the natural variability of the coastal system.

The objective of this study is to assess the applicability and limitations of a computer-based approach to calibrate the GenCade model. A simple method is employed to quantify model input parameter uncertainty. The PE and UA are applied to the three calibration parameters in GenCade: $K_{1}, K_{2}$, and $I S M O O T H$, which regulate the magnitude of the sand transport $\left(K_{1}\right.$ and $\left.K_{2}\right)$ and the smoothing of the bathymetry contour for wave refraction (ISMOOTH). Based on application experience, these three parameters are considered to be the most significant in calibrating the GenCade model (Frey et al. 2014). However, the computer-based calibration methodology profiled 
herein may be applied with any number of calibration parameters. This CHETN documents the methodology used and presents the results for an idealized case study and a field application. The methodology is discussed and recommendations are made regarding its applicability to the other model calibration parameters.

\section{BACKGROUND}

GenCade Calibration. The current state of the practice is to calibrate GenCade in two phases. First, the user manually calibrates parameters that affect the entire GenCade grid (referred to as global parameters). These parameters include the $K_{1}$ and $K_{2}$ coefficients of the longshore sand transport (LST) equation and the ISMOOTH value, which relates to the smoothing of the offshore bathymetric contour for wave refraction. These parameters cannot be measured and must be determined during the calibration process. Second, the user may adjust other sets of parameters that have a physical meaning but where the theoretical value is often unknown. These parameters relate to local features of the grid such as inlets and structures (e.g., the bypassing coefficient of the updrift inlet boundary, the jetty porosity, the attachment bar width and location and the shoal volumes). While the modeler has an educated idea of the range of possible values for these parameters, their final value may also be determined by trial and error. A thorough description of the GenCade model calibration parameters and how they affect model simulation can be found in Frey et al. (2014).

To demonstrate the automated calibration methodology, only the GenCade global model input parameters are specified as adjustable. The $K_{1}$ and $K_{2}$ are empirical coefficients present in the equation to calculate the LST rate $\left(\mathrm{m}^{3} / \mathrm{sec}\right)^{*}$ in GenCade. The LST equation is based on the extended version of the classical "CERC" ${ }^{\prime}$ relation developed by Kraus and Harikai (1983) and described in Frey et al. (2012b, page 11). The $K_{l}$ parameter is a coefficient that appears in the breaking wave angle term, and its value impacts the magnitude of the calculated transport. The $K_{2}$ coefficient appears in the derivative of the breaking wave height with respect to the alongshore axis term and is most significant in areas where there is sudden variation in breaking wave height such as near a structure. In past GenCade projects, values for $K_{l}$ ranged from 0.5 to 0.8 and values for $K_{2}$ ranged from 0.2 to 0.5 .

The offshore wave parameters are transformed to the breaking point using an offshore contour. The offshore contour (not to be confused with the regional contour) is a smoothed version of the shoreline at each time-step. ISMOOTH is the number of cells used in the smoothing window applied to the shoreline to produce the offshore contour and must be an odd integer number greater than 1 . One can argue that it is not a calibration parameter since this value should be set to the smallest value that leads to stable results, similar to the way the modeler chooses the simulation time-step. However, from experience in applying GenCade, it has been found that ISMOOTH acts as a "spatial diffusivity coefficient," sometimes slowing down erosion of large features when set to a large value or sustaining steep gradient in shoreline orientation when set to small value.

\footnotetext{
${ }^{*}$ For a full list of the spelled-out forms of the units of measure used in this document, please refer to US Government Publishing Office Style Manual, 31st ed. (Washington, DC: US Government Publishing Office 2016), 248-52, https://www.govinfo.gov/content/pkg/GPOSTYLEMANUAL-2016/pdf/GPO-STYLEMANUAL-2016.pdf.

${ }^{\dagger} \mathrm{CERC}=$ Coastal Engineering Research Center
} 
ISMOOTH was therefore included in the automated calibration to determine if it would converge toward a value that was similar to the manually calibrated value. More detailed discussion of the governing equations and the role of the calibration parameters can be found in Frey et al. 2012b and 2014, respectively.

Model Uncertainty. While there are only a few examples of model uncertainty analysis of shoreline change models (Ruggiero et al. 2010; Qiu 2013), there exists a vast amount of related work in the fields of hydrology and meteorology (Doherty [2015]; Muleta and Nicklow [2005]; Mishra [2009]; Skahill [2013]; and references cited within those references). The role of UA is to describe the entire set of possible outcomes with their associated probability of occurrence (Loucks et al. 2005). UA is a very broad topic both in the complexity of quantifying those uncertainties and in the multitude of methods available to calculate the propagation of those uncertainties.

A review of the important terms and concept of PE and UA is presented by Sánchez et al. (2015). Types of uncertainties and methods for quantifying them is briefly presented here. Uncertainties can be separated into two groups: epistemic and aleatory. Epistemic uncertainties can be reduced by increasing the knowledge or understanding of the system and improving its mathematical representation. Aleatory or stochastic uncertainties arise from the natural variability of the system and cannot be reduced. The model parameter uncertainties include uncertainties of the model input parameters (berm height, $K_{l}$ and $K_{2}$, shoreline position, etc.), forcing (accuracy of wave data) and measurements (measured shoreline position, measured longshore transport rate). The model parameter uncertainties are epistemic and can be reduced by better data collection and quality control. Another type of epistemic uncertainty is the model structural uncertainties. Model structural uncertainty stems from the imperfect mathematical representation of the processes by the model. With application of GenCade significant uncertainties are due to the natural variability of the coastal system, both spatially and temporally. An important source of GenCade model output uncertainties is the variability of the input wave data. Although the natural variability of the system cannot be reduced, it can certainly be quantified, and the magnitude might be, in some cases, even larger than the model parameter uncertainties. For example, Frey et al. (2012) found that climate variability had a large impact on regional sand transport with mean yearly transport changing direction from year to year.

Model prediction error will largely depend on the uncertainty of the most sensitive adjustable model parameters. If an insensitive parameter is also highly uncertain, then the modeler should not spend too much effort to try to reduce or quantify this uncertainty. For example, GenCade is very sensitive to the wave direction and in most cases not as sensitive to the $K_{2}$ parameter. Therefore, more effort should be taken in selecting quality wave data than in trying to reduce the uncertainty of the $K_{2}$ parameter.

The second aspect of quantifying model uncertainty relates to the method used. There exist two broad approaches to the quantification of uncertainty: Bayesian approach (full distribution analysis) and frequentist approach such as Classical Monte Carlo and first and second moment analysis (Gallagher and Doherty 2007; Willink and White 2012). Review and further references are provided in Sánchez et al. (2015). In this particular study, a frequentist PE and UA approach was chosen because it provides a simple method to determine the model parameter uncertainty. 
METHODOLOGY: The method employed for the automated calibration and uncertainty analysis of GenCade parameters $K_{l}, K_{2}$ and ISMOOTH is presented below.

Parameter Estimation. The goal of the optimization algorithm used during PE is to minimize an objective function (OF) while varying the calibration parameter(s). The OF is typically a goodness-of-fit statistic between the calculation performed with a set of parameters and the measurements. Depending on the value obtained for the OF, the algorithm will then determine new parameter values, execute the model, and recalculate the OF until a minimum is reached. There are several measures of goodness-of-fit statistics that have been used successfully with GenCade to quantify the agreement between measurement and calculation for example: correlation coefficient, mean absolute error (MAE), root mean square error, bias and Brier Skill Score (BSS). Interestingly, good scores for correlation coefficient and MAE do not necessarily translate to shoreline comparison results that are visually appealing (Szmytkiewicz et al. 2000). Through experience gained comparing shoreline data, it has been found that the BSS is a good indicator of the skill of the GenCade model. The BSS is defined as follows:

$$
B S S=1-\frac{\sum\left(x_{m}-x_{c}\right)^{2}}{\sum\left(x_{r e f}-x_{c}\right)^{2}}
$$

where $x_{m}$ is the measured shoreline position with respect to the grid, $x_{c}$ is the calculated shoreline, and $x_{r e f}$ is a reference shoreline in this case, the initial shoreline. The BSS ranges from negative infinity to 1. A performance score of 1 indicates a perfect agreement between measured and calculated values. Scores equal to or less than 0 indicate that the initial shoreline (reference shoreline) is a better predictor than the calculated values. Because the BSS needs to be minimized, the OF was defined as $1-B S S$ which is also referred to as the Normalized Mean Square Error (Qiu 2013).

The algorithm requires the user to supply a starting point for the parameters. The closer the initial guess is from the true value, the faster the algorithm will converge. Here, the optimization algorithm used is an unconstrained, multivariable, derivative-free method called the Nelder-Mead simplex method (Lagaris 1998). As the name suggests, the algorithm solves for several parameters at once. Parameter constraints were added by imposing penalty functions within the OF if the values are outside predetermined bounds. The penalty was set to increase linearly as it approaches the bound. These bounds are user specified and can be changed depending on the specifics of a project. Bounds for the application presented in this document were set to [0,1] for $K_{1}$ and $K_{2}$ and to $[1,100]$ for ISMOOTH. Another difficulty arises from the treatment of ISMOOTH, which is an odd integer number. The GenCade executable automatically rounds up to the nearest odd number when the user supplies a real value; for example, a value of 9.54 will be changed to 11 . Therefore, for the optimization algorithm, an ISMOOTH value of 9.5 and 10.5 will lead to the same OF number, making it more difficult to converge. The optimization algorithm was modified so that the step value for the next ISMOOTH test point is large enough to allow the algorithm to explore wide range of values. This large step value avoids the problem with the algorithms applying ISMOOTH values that are too close to each other, resulting in the same OF number.

Uncertainty Analysis. For this study, the frequentist UA approach described in Gallagher and Doherty (2007) was selected. This non-linear error analysis is sometimes referred to as the First- 
order Second-moment Method (Dettinger and Wilson 1981). It consists of propagating the various input variance through the modeling system to obtain the model output uncertainty. Once the parameters are determined via PE, the next step is to calculate the standard deviation of the residuals $\left(\sigma_{r}\right)$ of the model results (shoreline position) calculated with the estimated optimal parameters $\left(x_{c}\right)$ and the observed data $\left(x_{m}\right)$ (shoreline position).

$$
\sigma_{r}=\sqrt{\frac{\sum\left(x_{m}-x_{c}\right)^{2}}{n-m}}
$$

where $n$ the number of measurements (length of vector $x_{m}$ ) and $m$ is the number of parameters estimated. The next step is to calculate the covariance matrix $C(p)$ of a set of parameters $p$ which for a non-linear model is given by the following:

$$
C(p)=\sigma_{r}^{2}\left(J^{t} W J\right)^{-1}
$$

where $J$ is the Jacobian matrix in which each column is composed of the derivative of every element of $x_{c}$ with respect to each parameter. For example, in the case where the set of parameters $p$ includes $K_{l}, K_{2}$ and ISMOOTH, the Jacobian matrix would be a $3 \times n$ matrix where each column is the partial derivative of the calculated shoreline with respect to $K_{1}, K_{2}$ and ISMOOTH, respectively. If there is noise in the measurements (i.e., measured shoreline), $\mathrm{W}$ is the inverse of a user-supplied covariance matrix of the measurement error (Doherty 2015). Since the methodology was developed using noiseless idealized case, this term was ignored. The diagonal elements of $C(p)$ denoted by $\sigma_{i}^{2}$ describe the variance of individual estimated parameters. Finally, the interval within which the true parameter lies at a level of confidence given by $1-\alpha$, which can by calculated by (Draper and Smith 1998):

$$
\operatorname{Prob}\left\{p_{i}-t_{\alpha / 2}(n-m) \sigma_{i} \leq p_{i} \leq p_{i}+t_{\alpha / 2}(n-m) \sigma_{i}\right\}=1-\alpha
$$

where $p_{i}$ is the estimated true value of the $i^{t h}$ parameter and $t_{\alpha / 2}(n-m)$ is the upper $\alpha / 2$ confidence point of the Student's $t$ distribution with $n-m$ degrees of freedom. Since the degree of freedom is very large, the Student's $t$ distribution approaches that of a Gaussian distribution. In this case, the $t_{\alpha / 2}(n-m)$ term is 1.96 for a $95 \%$ confidence interval (or $\alpha=0.05$ ). This confidence interval represents the level of uncertainty pertaining to the calibration of the model parameters and does not necessary translate to the confidence level when the model is in prediction mode (Louckes et al. 2005).

APPLICATION: A simple idealized GenCade model test case with known model parameter values was created to verify application of the employed PE and UA methodology. The methodology was subsequently validated using a simple field application.

Idealized Case. The PE analysis requires the GenCade model to be run approximately 10 to 50 times, so an idealized test case was designed to run relatively quickly while still retaining typical features encountered in a field application. The idealized test case (shown in Figure 1) consists of a curved shoreline $3.6 \mathrm{~km}$ long with two $160 \mathrm{~m}$ long diffracting groins. The 360 grid cells are each 10 $\mathrm{m}$ wide, and the wave input was set to a constant swell with waves $0.75 \mathrm{~m}$ high and an $8 \mathrm{sec}$ period approaching the shore-normal at $-15 \mathrm{deg}$ relative to shore normal. The simulation was run for 1 year 
with calibration parameters set to $K_{l}=0.7, K_{2}=0.3$, and $I S M O O T H=11$. This simulation was saved and the calculated shoreline was used to represent the true or measured values.

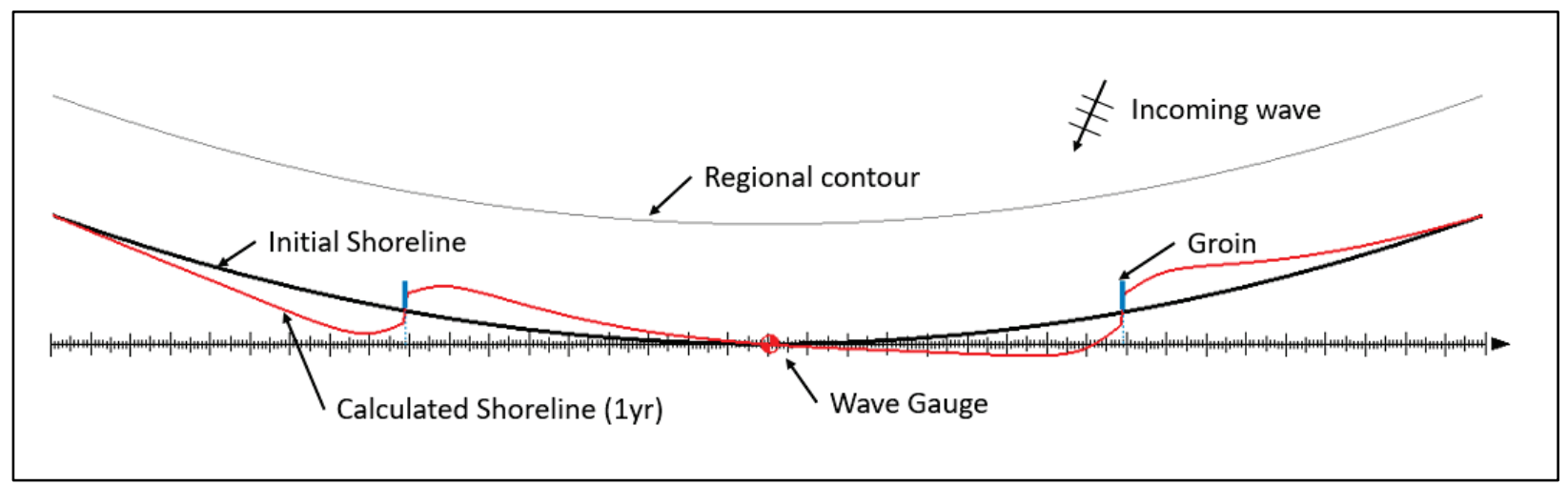

Figure 1: GenCade grid of idealized test case.

The parameter estimation was applied to the idealized case. Initial parameters of $K_{1}=0.5, K_{2}=$ 0.25 and $I S M O O T H=21$ were provided as a starting point for the optimization algorithm. The routine took 56 iterations for a total run time of $16 \mathrm{~min}$ (on a personal computer) to find the optimal calibration parameters (i.e. $K_{l}=0.7, K_{2}=0.3$, and $I S M O O T H=11$ ). Once the PE was completed, the Jacobian matrix of the partial derivative was produced for the calculation of the confidence interval. It was found that the calibrated value matches the true value almost perfectly with a standard error on the order of $10^{-4}$. The resulting confidence interval is smaller than the significant digits of the parameter. The model is not sensitive to variation of parameters smaller than the second decimal. The BSS for the final iteration is 1.0000 .

Field Case. The field application was extracted from the grid of a larger GenCade model application for Onslow Bay, NC (Frey et al. 2012a). The selected region consists of a developed barrier island known as Topsail Island. The grid is $27 \mathrm{~km}(17 \mathrm{mi})$ long and consists of 295 cells,

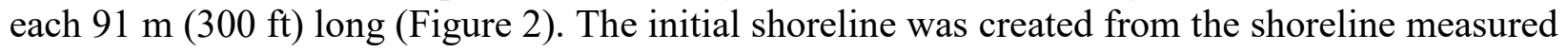
in 2004. For simplicity, a region was selected with no structures or inlets. The 2009 shoreline was used as the final measured shoreline for calibration. Wave information for the 2004 to 2009 calibration period was extracted from two wave gauges from the 2014 reprocessing of the Wave Information Study (WIS v_03) (USACE 2014). 


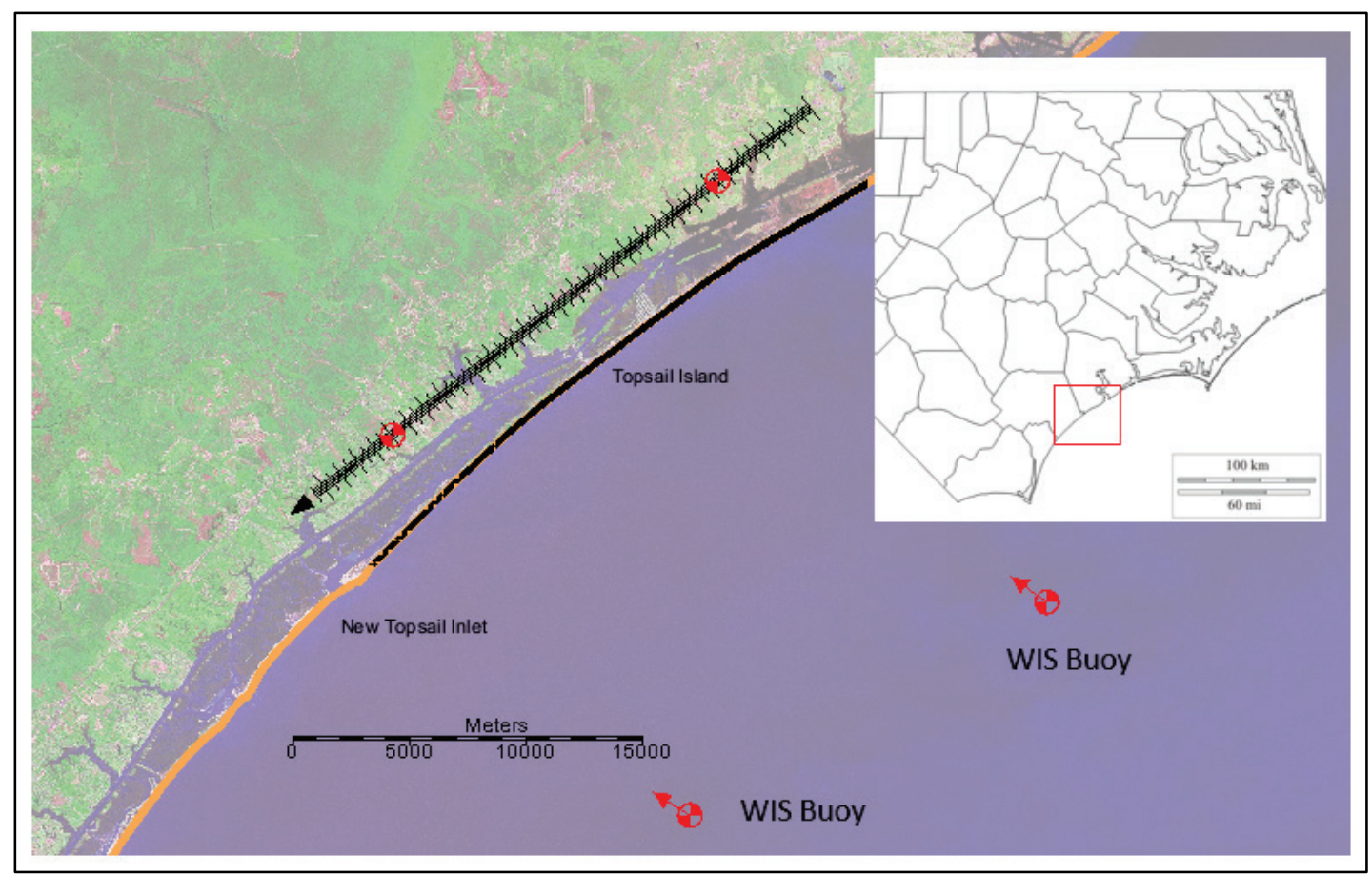

Figure 2. Onslow Bay, NC, GenCade grid utilized for PE and UA analysis.

Sensitivity analysis of the field case with the three global calibration parameters shows that the shoreline position is not sensitive to the $K_{2}$ parameter, which is to be expected since there are no structures in the grid. Preliminary testing of PE with $K_{2}$ caused the model to not converge since any value of $K_{2}$ produced the same results. The $K_{2}$ parameter was therefore removed from the automated calibration routine and set to the default value of 0.25 . Initial parameters of $K_{l}=0.6$ and $I S M O O T H=21$ were provided as the starting points for the optimization algorithm. The parameter values determined during $\mathrm{PE}$ as well as the standard error and $95 \%$ confidence interval are presented in Table 1. Table 1 also shows the parameter values that were chosen by the modeler during the manual calibration of the larger GenCade project. The PE routine took 68 iterations for a total run time of $31 \mathrm{~min}$ to converge. The BSS obtained at the last iteration was 0.59 .

\section{Table 1. Result of PE and UA for the field case.}

\begin{tabular}{|c|c|c|c|c|c||}
\hline \hline Parameters & $\begin{array}{c}\text { Value from } \\
\text { Manual } \\
\text { Calibration }\end{array}$ & Starting Point & $\begin{array}{c}\text { Value from } \\
\text { Automated } \\
\text { Calibration }\end{array}$ & $\begin{array}{c}\text { Standard } \\
\text { Error }\end{array}$ & $\begin{array}{c}\text { Lower and Upper } \\
\text { bound of 95\% } \\
\text { conf. int. }\end{array}$ \\
\hline K1 & 0.6 & 0.6 & 0.49 & 0.034 & $0.42-0.55$ \\
\hline ISMOOTH & 21 & 21 & 11 & 4 & $3-17$ \\
\hline
\end{tabular}

Figure 3 shows the shoreline change calculated using the optimum parameters for $K_{1}$ and ISMOOTH determine during $\mathrm{PE}$ as well as the measured shoreline change. The shoreline change is equal to the final shoreline position minus the initial shoreline position. The model was then rerun with the upper and lower bound for each parameter and a maximum and minimum envelop was constructed to represent the $95 \%$ confidence intervals. The dashed lines represent the intervals within which the true value of $K_{l}$ and ISMOOTH lies with a $95 \%$ confidence. 


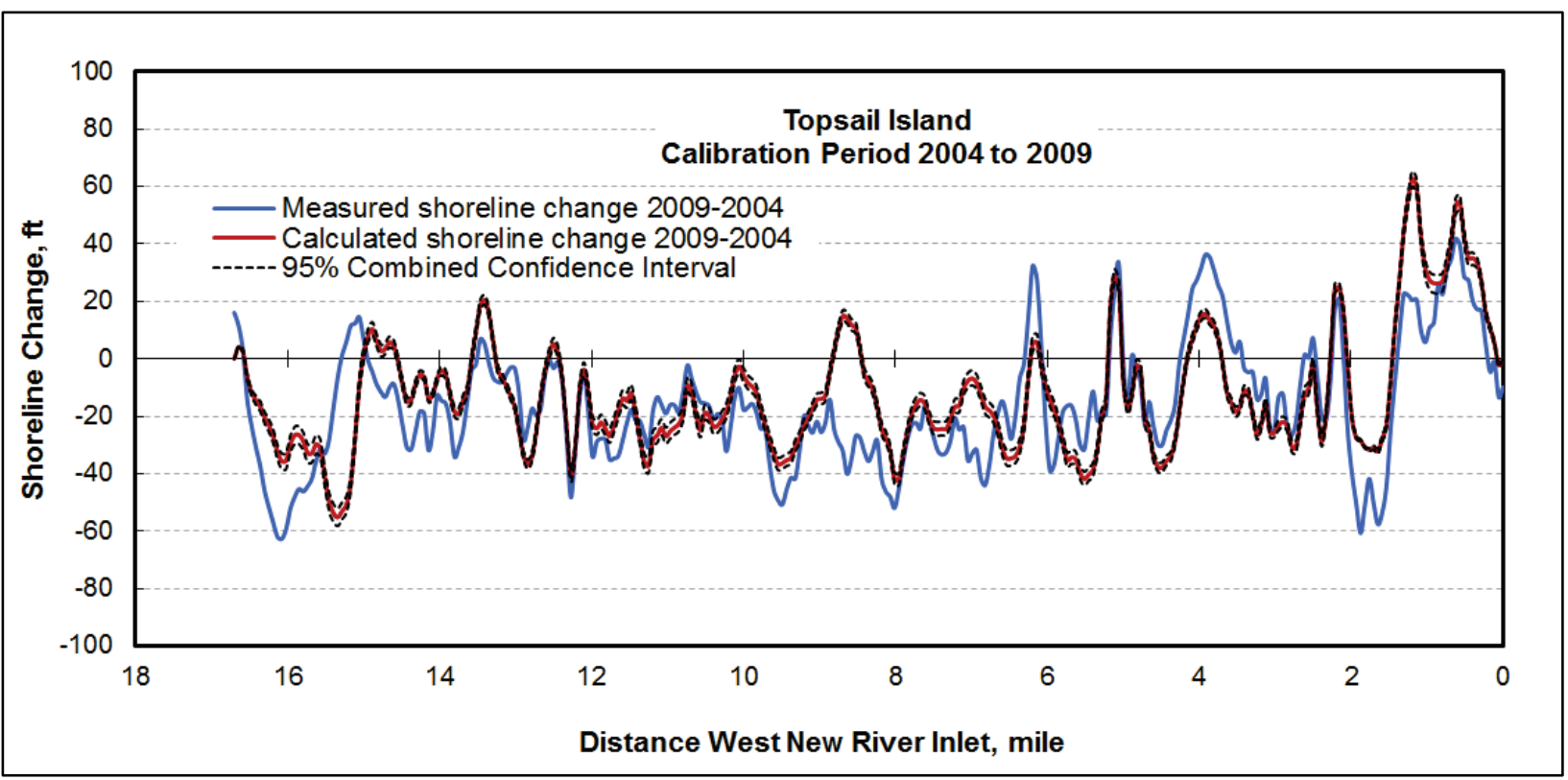

Figure 3. Calculated vs. measured shoreline changes using the optimum calibration parameters.

DISCUSSION: For the field application, the variance of the individual calibration parameters and the corresponding confidence intervals are relatively small when compared to the shoreline variability. This indicates that the variance of calibration parameters is only a small fraction of the overall predictive uncertainty of the model. Structural model uncertainty and climate variability are expected to contribute more significantly to uncertainty and should therefore be carefully considered in subsequent work on predictive model uncertainty quantification. Another alternative might be to steer GenCade toward a more data-driven approach such as the one utilized by Ruggiero et al. (2010) and more recently by Wang and Reeve (2010) and Alvarez and Pan (2016). These approaches use a realistic range of possible input conditions resulting in a range of possible future conditions rather than specific shoreline change predictions.

The automated calibration successfully determines optimum calibration parameter in the two cases presented. The robustness of the algorithm was tested by using different initial estimates as the starting value for the parameter. In all cases, the algorithm converges to the same answers. In general, the $K_{2}$ parameter only influences the shoreline position in small areas near a structure and should not be included in the PE unless the grid contains such structures. The method was also successful in calibrating the ISMOOTH parameter although the value was treated as a real number by the optimization algorithm and UA. Future implementation of PE and UA in GenCade should include the use of a spatial weighting factor where the user could exclude or reduce the importance of a section of the grid where the model is not expected to perform well such as inside the inlet throat or near inlet bank. The weighting factor would also be needed if the method was to expand to the other local calibration parameters. This study employed simple methods for PE and UA and future work should focus on more comprehensive methods that could accommodate the integer number issues and the natural variability of the system.

CONCLUSION: This study was aimed at determining if simple PE and UA could be implemented successfully in GenCade. The automated calibration routine developed was able to determine the optimum values of the global parameter $K_{1}, K_{2}$ and ISMOOTH in two simple test cases. The 
methods could be applied to larger more complex GenCade grids and to local calibration parameters with the use of a spatial weighting factor. The parameter uncertainty determined via non-linear variance analysis of the calibration parameters seems to be a small contributor to the overall model uncertainty. It is recommended that a thorough analysis of the sources of uncertainties should be performed and more research on the use of probabilistic approaches to quantify model uncertainty should be conducted.

AVAILABILITY: The GenCade executable is available through the Coastal Inlets Research Program (CIRP) website (http://cirp.usace.army.mil/products) under Products - GenCade.

Additional Information: This CHETN was prepared as part of the CIRP and was written by Sophie Munger (Sophie.Munger@gmail.com) of Blue Science Consultants. Dr. Sung-Chan Kim ([Sung-Chan.Kim@,usace.army.mil], voice: 601-634-3783) of the US Army Engineer Research and Development Center (ERDC), Coastal and Hydraulics Laboratory (CHL), leads GenCade development. Dr. Yan Ding, Dr. Brian Skahill, and Ashley Frey provided peer review of this publication. This technical note should be referenced as follows:

Munger, S. 2020. Computer-Based Calibration and Uncertainty Analysis of GenCade:

Description and Proof of Concepts. ERDC/CHL CHETN-IV-126. Vicksburg, MS: US

Army Engineer Research and Development Center. http://dx.doi.org/10.21079/11681/37900

\section{REFERENCES}

Alvarez, F., and S. Pan. 2016. "Predicting Coastal Morphological Changes with Empirical Orthogonal Function Method." Water Science and Engineering 9(1): 14-20. https://www.sciencedirect.com/science/article/pii/S167423701600003X\#!

Dettinger, M. D., and J. L. Wilson. 1981. "First Order Analysis of Uncertainty in Numerical Models of Groundwater Flow Part: 1. Mathematical Development." Water Resour. Res. 17(1): 149-161.

Doherty, J. 2015. Calibration and Uncertainty Analysis for Complex Environmental Models. Brisbane Australia: Watermark Numerical Computing. ISBN: 978-0-9943786-0-6.

Draper, N. R., and M. Smith. 1998. Applied Regression Analysis. Hoboken, NJ: John Wiley and Sons, Inc.

Frey, A. E., D. B. King, and S. Munger. 2014. Recommendation and Requirements for GenCade Simulations. ERDC/CHL TR-14-6. Vicksburg, MS: US Army Engineer Research and Development Center.

Frey, A. E., S. Munger, G. L. Williams, M. J. Wutkowski, and K. B. Conner. 2012a. GenCade Application at Onslow Bay, North Carolina. ERDC/CHL CHETN-I-85. Vicksburg, MS: US Army Engineer Research and Development Center.

Frey, A. E., K. J. Connell, H. Hanson, M. Larson, R. C. Thomas, S. Munger, and A. Zundel. 2012b. GenCade Version 1 Model Theory and User's Guide. ERDC/CHL TR-12-25. Vicksburg, MS: US Army Engineer Research and Development Center.

Gallagher, M., and J. Doherty. 2007. "Parameter Estimation and Uncertainty Analysis for a Watershed Model." Environmental Modeling \& Software 22(7): 1000-1020.

Kraus, N. C., and S. Harikai. 1983. "Numerical Model of the Shoreline Change at Oarai Beach." Coastal Engineering 7(1): 1-28. 
Lagaris, J. C., J. A. Reeds, M. H. Wright, and P. E. Wright. 1998. "Convergence Properties of the NelderMead Simplex in Low Dimensions.” SIAM Journal on Optimization 9(1): 112-147.

Loucks, D. P., E. Van Beek, J. R. Stedinger, J. P. Dijkman, and M. T. Villars. 2005. Water Resources Systems Planning and Management: An Introduction to Methods, Models, and Applications. Paris: Unesco.

Mishra, S. 2009. "Uncertainty and Sensitivity Analysis Techniques for Hydrologic Modeling.” Journal of Hydroinformatics 11(3-4): 282-296.

Muleta, M. K., and J. W. Nicklow. 2005. "Sensitivity and Uncertainty Analysis Coupled with Automatic Calibration for a Distributed Watershed Model." Journal of hydrology 306(1): 127-145.

Qiu, L. 2013. Analysis of Model Performance Related to Uncertainty in the Model BEACHPLAN for the Simulation of Shoreline Evolution. MSc thesis. University of Southampton: Faculty of Engineering and the Environment.

Ruggiero, P., M. Buijsman, G. M. Kaminsky, and G. Gelfenbaum. 2010. "Modeling the Effects of Wave Climate and Sediment Supply Variability on Large-Scale Shoreline Change." Marine Geology 273(14): $127-140$.

Sánchez, A., B. E. Skahill, W. Scharffenberg, and C. H. Smith. 2015. Computer-Based Model Calibration and Uncertainty Analysis: Terms and Concepts. ERDC/CHL CHETN-I-86. Vicksburg, MS: US Army Engineer Research and Development Center.

Skahill, B. E. 2013. Practice Driven and State-of-the-Art Methods to Quantify Hydrologic Model Uncertainty. ERDC/CHL CHETN-IV-87. Vicksburg, MS: US Army Engineer Research and Development Center.

Szmytkiewicz, M., J. Biegowski, L. M. Kaczmarek, T. Okroj, R. Ostrowski, Z. Pruszak, G. Różyńsky, and M. Skaja. 2000. "Coastline Changes nearby Harbour Structures: Comparative Analysis of OneLine Models versus Field Data.” Coastal Engineering 40(2): 119-139.

USACE (US Army Corps of Engineers). 2012. Wave Information Studies. Accessed September 17. http://wis.usace.army.mill

Wang, B., and D. Reeve. 2010. "Probabilistic Modeling of Long-Term Beach Evolution near Segmented Shore-Parallel Breakwaters." Coastal Engineering 57(8): 732-744.

Willink, R., and R. White. 2012. Disentangling Classical and Bayesian Approaches to Uncertainty Analysis. Technical Report No. CCT/12-08.

NOTE: The contents of this technical note are not to be used for advertising, publication, or promotional purposes. Citation of trade names does not constitute an official endorsement or approval of the use of such products. 